\title{
Perinatal Anemia is Associated with Neonatal and Neurodevelopmental Outcomes in Infants with Moderate to Severe Perinatal Asphyxia
}

\author{
Willemien S. Kalteren Hendrik J. ter Horst Anne E. den Heijer \\ Leanne de Vetten Elisabeth M.W. Kooi Arend F. Bos \\ Department of Pediatrics, Division of Neonatology, Beatrix Children's Hospital, University of Groningen, University \\ Medical Center Groningen, Groningen, The Netherlands
}

\section{Keywords}

Perinatal anemia $\cdot$ Hypoxic-ischemic encephalopathy ·

Mortality $\cdot$ Neurodevelopmental outcome

\begin{abstract}
Background: Perinatal anemia may cause perinatal asphyxia. Its pathophysiology and neurodevelopmental effects are theoretically different from other causes of perinatal asphyxia. Objective: The study aimed to determine whether perinatal anemia results in different short-term and long-term outcomes than other causes of perinatal asphyxia treated with therapeutic hypothermia. Methods: We retrospectively included infants with moderate to severe hypoxic-ischemic encephalopathy, born between May 2009 and October 2015. During follow-up, we assessed cognitive and motor development at 2-3 years of age, using the Bayley Scales of Infant and Toddler Development, third edition (BSID-III). Neurodevelopmental outcome (NDO) was classified as abnormal in case of cerebral palsy with Gross Motor Function Classification System $\geq$ III and/or a BSID-III composite score $<85$. Outcomes of infants with perinatal anemia (initial hemoglobin $<7 \mathrm{mmol} / \mathrm{L}$ ) were compared to infants born with perinatal asphyxia due to other causes. Results: In total, 111 infants were included
\end{abstract}

of whom 30 infants (27\%) died during the neonatal period. Infants with anemia $(n=23)$ had a higher mortality risk, OR $3.33,95 \% \mathrm{Cl} 1.27-8.72, p=0.01$. None of the surviving infants with anemia $(n=12)$ had an abnormal NDO, in contrast to 26/69 (38\%) with neurodevelopmental impairments, particularly motor problems, in the non-anemic group, $p<0.01$. Conclusions: Perinatal anemia causing moderate to severe perinatal asphyxia is associated with a higher risk for neonatal mortality. All survivors with perinatal anemia, however, showed a normal NDO in contrast to children who were born asphyxiated due to other causes. The underlying pathophysiological mechanism for the favorable NDO in the perinatal anemia group needs further elucidation.

$$
\begin{aligned}
& \text { ๑ } 2018 \text { The Author(s) } \\
& \text { Published by S. Karger AG, Basel }
\end{aligned}
$$

\section{Introduction}

In case of asphyxia, generally a combination of hypoxia or hypoxemia and/or reduced blood supply occurs [1]. As a result oxygen delivery is diminished, which can cause serious damage to the brain and other organs. Despite improvements in perinatal care, infants born after mod-

\begin{tabular}{ll}
\hline KARGER & $\begin{array}{l}\text { @ } 2018 \text { The Author(s) } \\
\text { Published by S. Karger AG, Basel }\end{array}$ \\
E-Mail karger@karger.com & This article is licensed under the Creative Commons Attribution- \\
www.karger.com/neo & $\begin{array}{l}\text { NonCommercial-NoDerivatives 4.0 International License (CC BY- } \\
\text { NC-ND) (http://www.karger.com/Services/OpenAccessLicense). } \\
\text { Usage and distribution for commercial purposes as well as any dis- } \\
\text { tribution of modified material requires written permission. }\end{array}$
\end{tabular}

Willemien S. Kalteren, BSc

Department of Pediatrics, Division of Neonatology, Beatrix Children's Hospital University of Groningen, University Medical Center Groningen

Hanzeplein 1, NL-9713 GZ Groningen (The Netherlands)

E-Mail w.s.kalteren@umcg.nl 
erate to severe asphyxia still suffer from short-term and long-term neurological sequelae. Children who survive are at higher risk for moderate to severe functional impairments [2]. In term neonates affected by hypoxic-ischemic encephalopathy (HIE) due to birth asphyxia, therapeutic hypothermia $(\mathrm{TH})$ reduces the risk of death or severe neurological sequelae [3].

One of the causes of moderate to severe perinatal asphyxia is perinatal anemia, mostly due to excessive blood loss. Earlier research demonstrated that a lower level of initial hemoglobin $(\mathrm{Hb})$ was a significant prognostic factor for abnormal neurodevelopmental outcome (NDO) in severely anemic asphyxiated infants [4].

After TH has been introduced, no studies on shortterm and long-term outcomes are available that distinguish perinatal anemia from other causes for moderate to severe perinatal asphyxia. Based on a small number of previous studies, we hypothesized a negative effect of low $\mathrm{Hb}$ concentrations in near-term and term asphyxiated infants on cognitive and motor development at 2-3 years of age.

\section{Methods}

\section{Participants and Clinical Parameters}

This was a retrospective cohort study, performed at the University Medical Center Groningen. The Medical Ethics Review Board of the University Medical Center Groningen approved this study. All infants, both inborn and outborn, that were diagnosed with moderate/severe perinatal asphyxia and treated with $\mathrm{TH}$ at our neonatal intensive care unit between May 2009 and October 2015, were included.

Perinatal asphyxia was clinically defined as an Apgar score (AS) of 5 or less at $5 \mathrm{~min}$ postpartum, either resuscitation/invasive ventilation for more than $10 \mathrm{~min}$, or umbilical cord $\mathrm{pH}<7.0$ and base deficit $>16 \mathrm{mmol} / \mathrm{L}$, or lactate $>10.0 \mathrm{mmol} / \mathrm{L}$. The attending neonatologist used the Thompson score to determine the severity of HIE. A score above 7 was considered as moderate to severe HIE and was used as cut-off value to start TH [5]. In case of an abnormal background pattern of the amplitude-integrated electroencephalography (aEEG), TH was also initiated.

Clinical data that we obtained from the infants' medical records included ASs, gestational age, birth weight, first $\mathrm{Hb}$ postpartum and $\mathrm{Hb} 24 \mathrm{~h}$ postpartum, aEEG background patterns during $\mathrm{TH}$, cerebral ultrasound, and MRI. Cerebral injury was determined on ultrasound and MRI. Cerebral ultrasound was considered abnormal in case of moderate to severe abnormalities (intraventricular/ periventricular hemorrhage grade 3-4, slit-like ventricles). MRI scans were assessed using the scoring system developed by Van Rooij et al. [6]. More extensive injury resulted in higher scores. The aEEG background patterns and EEG recordings were scored as normal (continuous normal voltage), mildly abnormal (discontinuous normal voltage, mildly epileptiform), or severely abnormal (burst suppression, continuous low voltage, flat trace, severely epileptiform) $[7,8]$.
We defined perinatal anemia as a $\mathrm{Hb}$ value below $7 \mathrm{mmol} / \mathrm{L}$ directly postpartum and/or $24 \mathrm{~h}$ after birth to account for acute perinatal blood loss that had not resulted in measurable anemia directly after birth. This cut-off value was chosen for practical reasons, as it was similar to the transfusion threshold of $7 \mathrm{mmol} / \mathrm{L}(11.3 \mathrm{~g} / \mathrm{dL})$ in our local protocol, which was adapted from our national guideline [9]. An $\mathrm{Hb}$ value of $7.0 \mathrm{mmol} / \mathrm{L}$ is more than 3 SDs below the mean, according to the reference values published in 1971 [10]. Next, we categorized the infants into 2 groups, those with perinatal anemia and those without (non-anemic). To assess the influence of perinatal anemia on neonatal outcome and on the child's neurodevelopment, we first compared the group of infants with perinatal anemia to the non-anemic group. Second, we investigated the effect of $\mathrm{Hb}$ value on NDO for the whole group, and for the 2 groups separately.

\section{Long-Term Follow-Up}

All infants who underwent $\mathrm{TH}$ and who survived were routinely tested at 2-3 years of age using the Bayley Scales of Infant and Toddler Development, third edition (BSID-III). The BSID-III assessment is a valid and commonly used tool, which examines the cognitive, language (receptive and expressive), and motor (gross and fine motor) abilities of children during infancy [11]. Cognitive and motor composite scores were determined. The standardized norm for each of the scales is 100 , SD 15 . We classified NDO as normal with BSID-III composite scores of $\geq 85$, and abnormal with a composite score $<85$.

Children diagnosed with cerebral palsy (Gross Motor Function Classification System $\geq$ III) or severe psychomotor retardation, were not invited for follow-up testing. They were classified as abnormal, based on information in their medical records.

Additionally, parents completed the Child Behavior Checklist (CBCL), a 100-item parent report that identifies behavioral problems of preschool children [12]. The items are converted into syndrome scale scores, from which internalizing and externalizing Tscores can be derived. The higher the T-scores, the more behavioral problems. Following the CBCL manual, we classified T-scores above 60 as borderline behavioral problems, above 63 as behavioral problems in the clinical range.

\section{Statistical Analysis}

We used IBM SPSS Statistics 23.0 (IBM Corp., Armonk, NY, USA) for statistical analyses. Patient characteristics were described as mean, SD or as median, interquartile range (IQR). The chisquare test was used to analyze the differences between groups. Next, we calculated ORs, including 95\% CIs for outcome. To adjust for potential confounders, we considered various parameters to include in multiple regression models, including AS at $5 \mathrm{~min}$, Thompson score, $\mathrm{pH}$ 1-h after birth, lactate concentration 1-h after birth, and postnatal age at start of active TH. We first checked whether these parameters were interrelated, because of multicollinearity, using Spearman's Rank Correlation test, in order to select the appropriate factors for potential inclusion in the multiple regression models. Of them, those factors that were univariately associated with mortality and NDO at $p<0.20$ were included in the multiple regression models. We also performed linear regression analyses to test whether $\mathrm{Hb}$ values were predictive for BSID-III composite scores. $p<0.05$ was considered to be statistically significant. 
Fig. 1. Flowchart inclusion and exclusion of the study population. Perinatal anemia was defined as a hemoglobin $(\mathrm{Hb})$ value below $7 \mathrm{mmol} / \mathrm{L}$ (11.3 mg/dL) directly after birth and/or $24 \mathrm{~h}$ after birth.

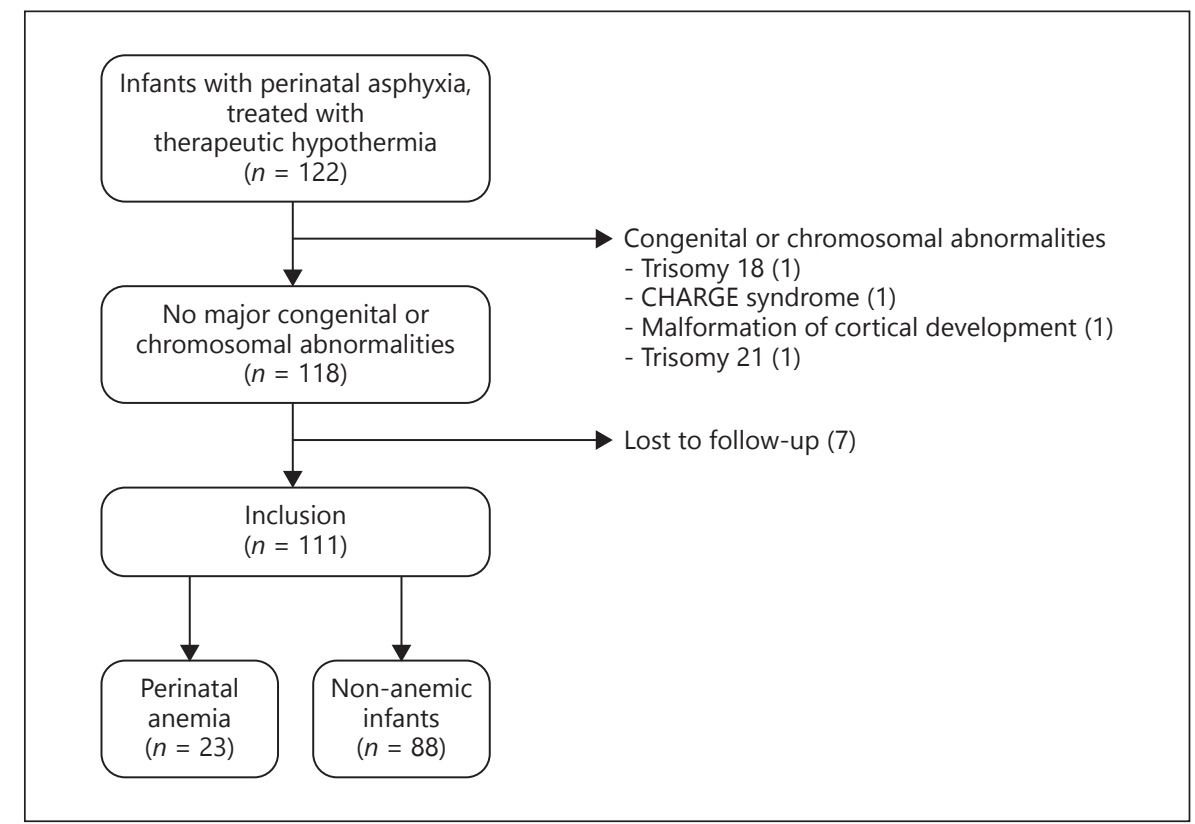

\section{Results}

\section{Clinical Data}

Of 122 eligible infants, we included 111 infants with a median gestational age of 39.86 weeks, IQR (38.2940.86). Four infants were excluded because of chromosomal abnormalities and 7 infants were lost to follow-up (Fig. 1).

The most frequent causes for perinatal asphyxia were prolonged labor (25\%), problems related to the umbilical cord (17\%), and placental abruption (14\%). In $22 \%$ of the cases, no etiology could be identified. We identified perinatal anemia in 23 infants (21\%), resulting in a range of initial $\mathrm{Hb}$ between 1.5 and $8.0 \mathrm{mmol} / \mathrm{L}$. Within this group, the most common causes were placental abruption (52\%), fetal-maternal transfusion (22\%), and umbilical cord rupture $(13 \%)$.

The majority ( $83 \%)$ of the anemic infants was born after a caesarean section, while this was $45 \%$ in the non-anemic group. Median AS and first $\mathrm{pH}$ postpartum were lower in anemic infants, but median Thompson scores and initial lactate concentrations did not differ between groups. Active TH was started at median postnatal age of $4 \mathrm{~h}$ and $30 \mathrm{~min}$. Brain MRI was performed at a median postnatal age of 7 days, IQR (6-8). MRI data was not available in 12 infants (11\%). Four of them died before MRI could be performed, and in 8 cases, MRI was clinically deemed unnecessary before redirection of care, based on isoelectric full EEG in combination with a clinical condition consistent with brain death. Clinical characteristics are presented in Table 1.

\section{Neonatal Outcome}

Thirty infants (27\%) died. Infants with anemia had a higher risk for mortality ( 48 vs. $22 \%$ ), OR $3.33,95 \%$ CI 1.27-8.72, $p=0.01$. After adjustment for potential confounders, AS 5th min and lactate $1 \mathrm{~h}$ after birth, the OR was $1.96,95 \%$ CI $0.63-6.11, p=0.25$. The extent of brain injury was investigated with the use of aEEG, EEG, and MRI. In those infants in whom MRI was performed, the degree of cerebral injury did not differ between groups (Table 1). Twenty-four infants died following a decision to redirect care due to the expected very poor prognosis based on clinical condition, MRI, and aEEG. Of those, $8(33 \%)$ had perinatal anemia. There was no difference in postnatal day of redirection of care or in the information on which we based the decision (Table 2).

\section{Neurodevelopmental Outcome}

At a median age of 25 months (IQR 24-30), the BSIDIII was performed in 75 children. All survivors in the severe perinatal anemia group $(n=12)$ had a normal outcome, whereas $26(38 \%)$ of the other patients had neurodevelopmental impairments at $2-3$ years of age, $p<0.01$ (Table 2). The BSID-III cognitive composite scores were comparable between the groups, while motor composite 
Table 1. Patient characteristics

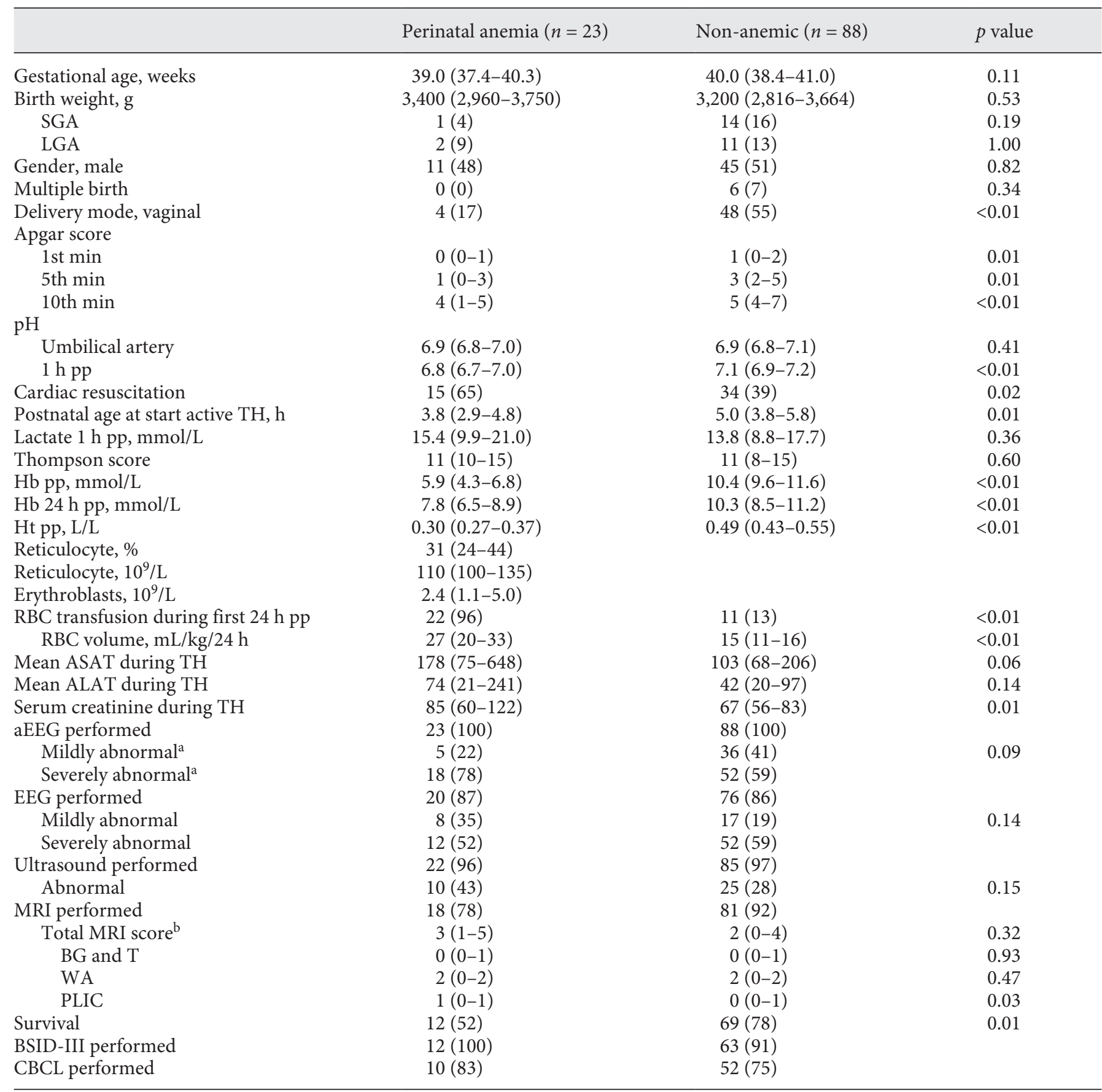

Data are presented as median (interquartile range) or as $n(\%)$.

SGA, small for gestational age (<p10); LGA, large for gestational age (>p90); TH, therapeutic hypothermia; Hb, hemoglobin; Ht, hematocrit; pp, postpartum; RBC, red blood cell; aEEG, amplitude-integrated electroencephalography; BSID-III, Bayley Scales of Infant and Toddler Development (3rd edition); CBCL, Child Behavior Checklist; BG and T, basal ganglia and thalamus; WA, watershed areas; PLIC, posterior limb of internal capsule.

${ }^{a}$ A mildly or severely abnormal background pattern at some point during therapeutic hypothermia.

b Total MRI score based on the scoring system developed by van Rooij et al. [6]. 
Table 2. Neonatal and neurodevelopmental outcomes

\begin{tabular}{lccc}
\hline & Perinatal anemia & Non-anemic & $p$ value \\
\hline Neonatal outcome, $n$ & 23 & 88 \\
Outcome & & & \\
$\quad$ Survival & $12(52)$ & $69(78)$ & 0.01 \\
$\quad$ Survival without disabilities & $12(52)$ & $43(49)$ & 0.82 \\
Redirection of care & $8(73)$ & $16(84)$ & 0.64 \\
Postnatal day redirection of care & $6(3-9)$ & $6(3-8)$ & 0.70 \\
Decision based only on aEEG and clinical condition & $5(63)$ & $7(44)$ & 0.67 \\
\hline Neurodevelopmental outcome & & & \\
BSID-III, $n$ & 12 & 69 & $<0.01$ \\
Normal & $12(100)$ & $43(62)$ & $<0.01$ \\
Abnormal & $0(0)$ & $26(38)$ & 0.98 \\
Cognitive composite score & $100 \pm 10$ & $100 \pm 12$ & 0.02 \\
Motor composite score & $104 \pm 11$ & $95 \pm 15$ & 0.61 \\
CBCL, $n$ & 10 & 52 & \\
Normal & $8(80)$ & $47(90)$ & $3(6)$ \\
Subclinical & $1(10)$ & $2(4)$ & 0.53 \\
Clinical & $1(10)$ & $44 \pm 10$ & 0.45 \\
Internalizing T-score & $43 \pm 7$ & $48 \pm 9$ &
\end{tabular}

Data are presented as $n(\%)$, median (IQR) or as mean \pm SD.

$\mathrm{Hb}$, hemoglobin; aEEG, amplitude-integrated electroencephalography; BSID-III, Bayley Scales of Infant and Toddler Development (3rd edition); CBCL, child behavior checklist.

scores were higher in the perinatal anemia group. We found no differences between groups in T-scores for behavioral problems (Table 2).

\section{$\mathrm{Hb}$ Values and Outcome}

In Table 3, we present ORs of initial $\mathrm{Hb}$ values and various potential confounders for adverse outcome, mortality, and abnormal NDO. After checking several variables on multicollinearity, we identified AS 5th min, lactate $1 \mathrm{~h}$ after birth, and postnatal age at start TH as appropriate to be entered in multivariate regression analyses as potential confounders. When including all infants in the analyses, initial $\mathrm{Hb}$ values were not associated with mortality and adverse outcomes (that is mortality and abnormal NDO), neither in univariate analyses, nor after adjustment for confounders (Table 3). When including only the 81 surviving infants in the analyses, higher initial $\mathrm{Hb}$ values increased the risk of abnormal NDO, which remained after adjustment for confounders, with OR $1.54,95 \%$ CI 1.14-2.07.

When we repeated the analyses in the 2 groups separately, ORs of $\mathrm{Hb}$ values for adverse outcomes and mortality differed considerably between anemic and nonanemic infants (Table 3). In anemic infants, initial $\mathrm{Hb}$ values were not associated with increased or decreased risk of adverse outcomes, but in non-anemic infants, higher initial $\mathrm{Hb}$ values increased the risk of adverse outcomes with OR 1.39, 95\% CI 1.03-1.86, after adjustment for confounders. In the 69 non-anemic survivors, higher initial $\mathrm{Hb}$ values also increased the risk of NDO with OR $1.35,95 \%$ CI $0.95-1.92$, but here the association just failed to reach significance.

Table 4 shows that initial $\mathrm{Hb}$ values were not significantly associated with cognitive composite scores or with $\mathrm{CBCL}$ results. Lower initial $\mathrm{Hb}$ values after birth resulted in better motor composite scores. In the non-anemic infants, we found a significant association between lower initial $\mathrm{Hb}$ and higher cognitive and motor composition scores.

\section{Discussion}

In this study, we demonstrate a higher risk for neonatal mortality in asphyxiated infants born with perinatal anemia. At the age of 2-3 years, however, survivors of perinatal anemia showed better NDOs than children who had perinatal asphyxia due to other causes. The degree of 
Table 3. ORs (95\% CI) of various risk factors in relation to neonatal and neurodevelopmental outcomes, using univariate and multiple regression analyses

\begin{tabular}{|c|c|c|c|}
\hline & All infants & Perinatal anemia & Non-anemic \\
\hline Adverse outcome, $n^{\mathrm{a}}$ & 111 & 23 & 88 \\
\hline \multicolumn{4}{|l|}{ Univariate regression analysis } \\
\hline $\mathrm{Hb} \mathrm{pp}, \mathrm{mmol} / \mathrm{L}$ & $1.09(0.94-1.25)^{\#}$ & $0.80(0.50-1.26)^{\#}$ & $1.40(1.04-1.87)^{*}$ \\
\hline Apgar score 5th min & $0.91(0.75-1.10)$ & $0.68(0.43-1.07)$ & $0.98(0.77-1.25)$ \\
\hline Lactate $1 \mathrm{~h}$ pp, mmol/L & $1.07(1.01-1.24)^{*}$ & $1.19(1.00-1.41)^{*}$ & $1.05(0.98-1.12)^{\#}$ \\
\hline PNA at start $\mathrm{TH}, \mathrm{h}$ & $1.10(0.86-1.41)$ & $2.02(0.93-4.38)$ & $0.98(0.73-1.30)$ \\
\hline \multicolumn{4}{|l|}{ Multiple regression analysis } \\
\hline $\mathrm{Hb} \mathrm{pp}, \mathrm{mmol} / \mathrm{L}$ & $1.11(0.96-1.29)$ & $0.90(0.53-1.53)$ & $1.39(1.03-1.86)^{*}$ \\
\hline Mortality, $n$ & 111 & 23 & 88 \\
\hline \multicolumn{4}{|l|}{ Univariate regression analysis } \\
\hline $\mathrm{Hb} \mathrm{pp}, \mathrm{mmol} / \mathrm{L}$ & $0.89(0.76-1.04)^{\#}$ & $0.80(0.50-1.26)^{\#}$ & $1.35(0.94-1.93)^{\#}$ \\
\hline Apgar score 5 th $\min$ & $0.62(0.48-0.81)^{*}$ & $0.68(0.43-1.07)^{\#}$ & $0.65(0.47-0.89)^{*}$ \\
\hline Lactate $1 \mathrm{~h} \mathrm{pp}, \mathrm{mmol} / \mathrm{L}$ & $1.11(1.03-1.19)^{*}$ & $1.19(1.00-1.41)^{*}$ & $1.09(1.00-1.18)^{*}$ \\
\hline PNA at start $\mathrm{TH}, \mathrm{h}$ & $0.98(0.74-1.29)$ & $2.02(0.93-4.38)$ & $0.92(0.65-1.30)$ \\
\hline \multicolumn{4}{|l|}{ Multiple regression analysis } \\
\hline $\mathrm{Hb} \mathrm{pp}, \mathrm{mmol} / \mathrm{L}$ & $0.97(0.81-1.15)$ & $0.88(0.51-1.53)$ & $1.34(0.91-1.96)$ \\
\hline Abnormal NDO, $n$ & 81 & $\mathrm{~b}$ & 69 \\
\hline \multicolumn{4}{|l|}{ Univariate regression analysis } \\
\hline $\mathrm{Hb} \mathrm{pp}, \mathrm{mmol} / \mathrm{L}$ & $1.53(1.14-2.07)^{*}$ & & $1.34(0.95-1.89)^{\#}$ \\
\hline Apgar score 5th min & $1.29(0.97-1.72)^{\#}$ & & $1.30(0.94-1.79)^{\#}$ \\
\hline Lactate $1 \mathrm{~h} \mathrm{pp}, \mathrm{mmol} / \mathrm{L}$ & $1.01(0.94-1.09)$ & & $1.01(0.93-1.09)$ \\
\hline PNA at start $\mathrm{TH}, \mathrm{h}$ & $1.17(0.86-1.59)$ & & $1.02(0.73-1.43)$ \\
\hline \multicolumn{4}{|l|}{ Multiple regression analysis } \\
\hline $\mathrm{Hb} \mathrm{pp}, \mathrm{mmol} / \mathrm{L}$ & $1.54(1.13-2.10)^{*}$ & & $1.35(0.95-1.92)$ \\
\hline
\end{tabular}

${ }^{*} p<0.05 ;{ }^{*} p<0.20$.

a Adverse outcome indicates death or abnormal neurodevelopmental outcome.

${ }^{\mathrm{b}}$ No infants with perinatal anemia had an abnormal NDO. Therefore, we did not perform separate logistic regression analyses for abnormal NDO in anemic infants.

NDO, neurodevelopmental outcome; Hb, hemoglobin; pp, postpartum; PNA, postnatal age; TH, therapeutic hypothermia.

Table 4. Relation between initial hemoglobin values after birth and BSID-III scores and CBCL T-scores, presented as B (95\% CI), in all surviving infants, and in surviving infants with and without perinatal anemia

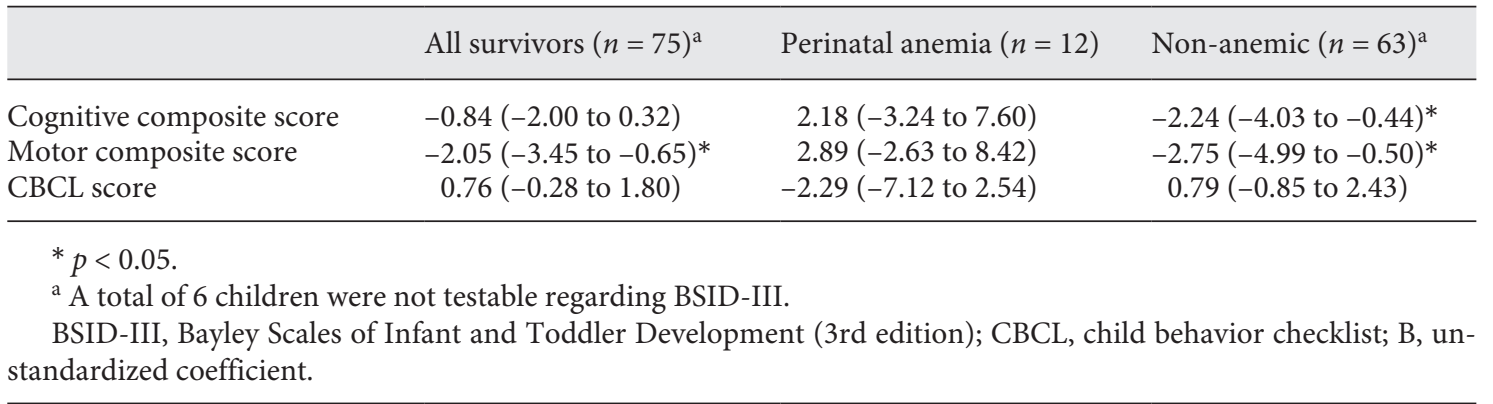

anemia was not predictive for NDO during infancy in anemic born infants.

A similar association between perinatal anemia and high neonatal mortality was found in 2 previous studies. In a study performed between 2000 and 2011, 18 (37\%) severely anemic patients died within $72 \mathrm{~h}$ after birth [13]. Muraskas et al. [14] also reported higher mortality rates in infants with perinatal anemia leading to birth asphyxia. They suggested that a marked reduction of blood flow to organs that are not essential for immediate survival had 
resulted in a higher incidence of multi-organ failure [14]. Severe anemia at birth may also have limited the oxygen delivery to vital organs in our anemic patients, attenuating the response to appropriate resuscitation. Lower AS and the more frequent need for cardiac resuscitation suggested a poorer condition after birth in the anemic group.

This higher mortality rate might be due to a difference in pathophysiology of injury between groups. Perinatal anemia leading to HIE might result in a more obvious pattern of caused injury than other causes for perinatal asphyxia. A possible explanation for the more clearly severe brain injury might be the length of the hypoxic-ischemic incident. Severe perinatal bleeding is an acute serious event, whereas other causes might be more prolonged less severe, hypoxic events. This short-lasting anemia might be more amenable to $\mathrm{TH}$ in terms of neuroprotection. Although, early initiation of TH might also be associated with a higher mortality rate in anemic infants. Perinatal anemia may thus lead to a clearer picture of the prognosis, which may affect the decision-making regarding expected serious long-term sequelae. In our patient population, however, we were not able to confirm these differences in neuroimaging or in decision-making.

In our cohort, survivors of perinatal anemia showed favorable NDOs at an age of 2-3 years. Kadooka et al. [4], however, previously demonstrated that severe anemia at birth resulted in more neurological sequelae. Infants in their study were not treated with TH though, which is known to significantly improve the outcome of asphyxiated infants [15]. Moreover, they did not report their rate of mortality, which may also have accounted for differences between their and our results. In line with our results, Zonnenberg et al. [13] showed normal NDOs in all surviving infants born with anemia and they suggested that neonatal anemia itself did not significantly affect the long-term outcome. Neurodevelopmental tests, however, were not routinely performed in their study.

The higher BSID-III scores might be explained by several theories. First, the inflammatory response following perinatal asphyxia highly contributes to post-HIE injury [16]. Following an acute, short hypoxic-ischemic incident, the inflammatory cascade might be less activated, but this is highly speculative. Second, anemic hypoxia during pregnancy and birth stimulates epo production, which might act as a protective mechanism against the adverse effects of asphyxia [17].

Third, the cut-off value of $7 \mathrm{mmol} / \mathrm{L}$ that we chose to define perinatal anemia may also account for these positive results, because it was slightly higher compared with other studies $[13,14]$. The anemic group may comprise

Outcomes in Anemic Asphyxiated Neonates some infants who did not suffer from severe perinatal anemia. An initial lower $\mathrm{Hb}$ concentration in severely anemic infants might result in higher risks for an adverse outcome. This association was not significant though, possibly due to the small sample size. On the contrary, lower $\mathrm{Hb}$ concentrations in the other group show a marked decrease for adverse outcome. Slightly lower Hb concentrations may improve organ perfusion by resulting in lower blood viscosity, which may in turn lead to an improved blood flow during hypoxia and hypoxemia, particularly in the cerebral microcirculation. This is a process similar to shock resuscitation in trauma care where they found a significant benefit in managing the blood viscosity, rather than the need to restore the oxygen carrying capacity [18]. A higher viscosity might also lead to worse aEEG patterns or more MRI abnormalities. We were, however, not able to confirm this in our study. Of note, none of our infants had abnormally high $\mathrm{Hb}$ values. Further studies are required to investigate the associations between $\mathrm{Hb}$ values, aEEG patterns, and MRI findings. Furthermore, anemia results in less oxygen carrying capacity to a potentially compromised brain. This may have led to less injury from free oxygen radicals, resulting in an improved long-term outcome. Based on these differing results of the associations between $\mathrm{Hb}$ concentrations and outcomes in the 2 groups, our results may reflect that infants with an $\mathrm{Hb}$ concentration postpartum around $7 \mathrm{mmol} / \mathrm{L}$ are in favor of positive outcomes following perinatal asphyxia.

We found significant associations between lower $\mathrm{Hb}$ concentrations and higher BSID-III motor composite scores in all surviving infants, but not for cognitive composite scores, possibly because of the age during followup testing. The children might have been too young for accurate testing of cognitive impairments. We also found no differences in scores for internalizing and externalizing behavioral problems. Behavioral outcomes of asphyxiated neonates have received little attention. Van Handel et al. [19] reported that children following HIE are at risk for behavioral problems, but their scores are mostly in the average range. Using questionnaires may also have accounted for not finding differences due to possible parental underestimation [20].

The present study has several limitations. First, because of the retrospective design, some information was limited in cases where parameters were not routinely determined. Second, we checked whether decisions regarding redirection of care differed between children with and without anemia, but were not able to find any differences. It is therefore not likely that redirection of care had influenced our results, but, being a retrospective study, we 
cannot fully exclude this possibility. Third, as this was a single center study, generalizability to other centers could be limited. The follow-up rate, however, was $92 \%$ which makes it a reliable representation. Fourth, different clinical etiologies of anemia may have had its own pathophysiological relation with outcome. The small sample size hampered us from performing subgroup analyses. Finally, the other causes for perinatal asphyxia include various etiologies for HIE. This may have caused bias, although we included a relatively large group of infants who had otherwise similar neonatal clinical conditions.

\section{Conclusion}

We have presented evidence that in the era of TH perinatal anemia remains associated with higher mortality rates compared to other causes of moderate to severe perinatal asphyxia. The anemic surviving infants, however, showed better NDOs during infancy compared to survivors without anemia. Furthermore, Hb concentrations may influence survival following HIE. This association between low and high $\mathrm{Hb}$ values after birth was striking and needs to be further elucidated.

Prospective studies with longer follow-up periods are needed to understand the pathophysiological differences and to identify possible interventions to improve neonatal care for these high-risk infants.

\section{Acknowledgments}

This study was part of the research program of the Graduate School of Medical Sciences, Research Institute SHARE, University of Groningen. Miss W.S.K. received financial support from the Junior Scientific Master Class of the University of Groningen.

\section{Disclosure Statement}

The authors declare no conflicts of interest.

\section{References}

1 Rainaldi MA, Perlman J: Pathophysiology of birth asphyxia. Clin Perinatol 2016;43:409422.

2 Ahearne CE, Boylan GB, Murray DM: Short and long term prognosis in perinatal asphyxia: an update. World J Clin Pediatr 2016;5: 67-74.

3 Jacobs SE, Berg M, Hunt R, Tarnow-Mordi WO, Inter TE, Davis PG: Cooling for newborns with hypoxic-ischaemic encephalopathy. Cochrane Database Syst Rev 2013: CD003311.

4 Kadooka M, Kato H, Kato A, Ibara S, Minakami H, Maruyama Y: Effect of neonatal hemoglobin concentration on long-term outcome of infants affected by fetomaternal hemorrhage. Early Hum Dev 2014;90:431-434.

5 Groenendaal F, Casaer A, Dijkman KP, Gavilanes AW, de Haan TR, ter Horst HJ, Laroche S, Naulaers G, Rijken M, van Straaten HL, Steiner K, Swarte RM, Zecic A, Zonnenberg IA: Introduction of hypothermia for neonates with perinatal asphyxia in the Netherlands and Flanders. Neonatology 2013;104:15-21.

6 Van Rooij LG, Toet MC, van Huffelen AC, Groenendaal F, Laan W, Zecic A, de Haan T, van Straaten IL, Vrancken S, van Wezel G, van der Sluijs J, Ter Horst H, Gavilanes D, Laroche S, Naulaers G, de Vries LS: Effect of treatment of subclinical neonatal seizures detected with aEEG: randomized, controlled trial. Pediatrics 2010;125:358-366.

7 Toet MC, Hellström-Westas L, Groenendaal F, Eken P, de Vries LS: Amplitude integrated
EEG 3 and $6 \mathrm{~h}$ after birth in full term neonates with hypoxic-ischaemic encephalopathy. Arch Dis Child Fetal Neonatal Ed 1999;81: F19-F33.

8 Hellström-Westas L, Rosén I, de Vries LS, Greisen G: Amplitude integrated EEG: classification and interpretation in preterm and term infants. Neoreviews 2006;7:e76-e87.

9 Von Lindern J, Brand A, Lopriore E: Revision of the guideline "blood transfusion": for the newborn. Ned Tijdscr Geneesk 2012;156: A4795.

10 Matoth Y, Zaizov R, Varsano I: Postnatal changes in some red cell parameters. Acta Paediatr Scand 1971;60:317-323.

11 Bruggink JL, Einspieler C, Butcher PR, Stremmelaar EF, Prechtl HF, Bos AF: Quantitative aspects of the early motor repertoire in preterm infants: do they predict minor neurological dysfunction at school age? Early Hum Dev 2009;85:25-36.

12 Achenbach TM, Rescorla LA: Manual for the ASEBA Preschool Forms \& Profiles. Burlington, BMJ Publishing Group, 2000, pp 11-14, 59.

13 Zonnenberg IA, Vermeulen RJ, Rohaan MW, van Weissenbruch MM, Groenendaal F, de Vries LS: Severe neonatal anaemia, MRI findings and neurodevelopmental outcome. Neonatology 2016;109:282-288.

14 Muraskas JK, Kelly AF, Nash MS, Goodman JR, Morrison JC: The role of fetal inflammatory response syndrome and fetal anemia in nonpreventable term neonatal encephalopathy. J Perinatol 2016;36:362-365.
15 Azzopardi D, Strohm B, Marlow N, Brocklehurst P, Deierl A, Eddama O, Goodwin J, Halliday HL, Juszczak E, Kapellou O, Levene M, Linsell L, Omar O, Thoresen M, Tusor N, Whitelaw A, Edwards AD; TOBY Study Group: Effects of hypothermia for perinatal asphyxia on childhood outcomes. N Engl J Med 2014;371:140-149.

16 Rocha-Ferreira E, Hristova M: Plasticity in the neonatal brain following hypoxicischaemic injury. Neural Plast 2016;2016: 4901014

17 Garg B, Sharma D, Bansal A: Systematic review seeking erythropoietin role for neuroprotection in neonates with hypoxic ischemic encephalopathy: presently where do we stand. J Matern Fetal Neonatal Med 2017:1-11.

18 Salazar Vázquez BY, Wettstein R, Cabrales P, Tsai AG, Intaglietta M: Microvascular experimental evidence on the relative significance of restoring oxygen carrying capacity vs. blood viscosity in shock resuscitation. Biochim Biophys Acta 2008;1784:1421-1427.

19 Van Handel M, Swaab H, de Vries LS, Jongmans MJ: Long-term cognitive and behavioral consequences of neonatal encephalopathy following perinatal asphyxia: a review. Eur J Pediatr 2007;166:645-654.

20 Van der Valk JC, van den Oord EJ, Verhulst FC, Boomsma D: Using parental ratings to study the etiology of 3-year-old twins' problem behaviors: different views or rater bias? J Child Psychol Psychiatry 2001; 42:921-931. 